\title{
Sistemas de labranza y densidades de la batata: calidad del suelo y de las raíces tuberosas ${ }^{1}$
}

\author{
Tillage systems and seeding densities in sweet potato: quality of soil and \\ reserve roots
}

\author{
Maiby Pérez-Darniz², Auris D. García-Méndez ${ }^{2}$,Marielena Medina ${ }^{2}$
}

\section{RESUMEN}

El objetivo de este trabajo fue determinar el efecto de sistemas de labranza y densidades de siembra en la calidad de las raíces tuberosas de batata (Ipomoea batata (L) Lamb.). El estudio se realizó en la Universidad Central de Venezuela, en el año 2014. Se determinaron los grados de severidad de la degradación del suelo y su correspondencia con las escalas de preferencia basadas en el peso unitario y dimensiones de la raíz tuberosa. Se usó un arreglo en franjas, donde los sistemas de labranza (SL) fueron asignados a las parcelas, y las densidades de siembra (Ds) a las franjas. Los SL consistieron en labranza vertical con cincel (LC), con arado de disco (LA), convencional con tres pases de rastra de disco (LCo) y mínima con un pase de rastra de disco (LM). Las Ds fueron de 0,25 cm (D1) y 0,15 cm (D2) en la hilera. La calidad física del suelo fue superior en los tratamientos de LA. De estos, la combinación con la distancia de siembra más larga (LA-D1) favoreció la mayor calidad postcosecha del cultivo. Al contrario, en los tratamientos con cincel se promovió la menor aceptación del consumidor. La ligera a moderada severidad de la degradación del suelo no explicó la variación de la calidad postcosecha. Se infiere sobre la menor tasa de movimiento de agua en LC, que promovió un exceso de humedad y la más baja calidad de la raíz tuberosa.

Palabras claves: labranza, raíz de reserva, propiedades del suelo.

\begin{abstract}
The aim of this work was to determine the effect of tillage systems and seeding densities in sweet potato (Ipomoea batata (L) Lamb.). The study was held on the Central University of Venezuela, in the year of 2014. The degrees of severity of soil degradation and its correspondence with the scales of preference based on unit weight and dimensions of the tuberous root were determined. An arrangement of slots was used, where the tillage systems (TS) were assigned to the parcels and the seeding densities (SD) were assigned to slots. The TS consisted on vertical farming with a chisel (LC), with disc plowing (LA), conventional with three passes of the disc harrow (LCo) and a minimum with a pass of disc harrow (LM). The Ds were of $0,25 \mathrm{~cm}$ (D1) and 0,15 $\mathrm{cm}$ (D2) in the line. The physical quality of the soil was superior in the LA treatments. In these, the combination with the longest planting distances (LA-D1) favored the highest postharvest quality. On the contrary, the chisel treatments promoted the least acceptances among consumers. The light to moderate severity of soil degradation did not explain the postharvest quality variation. It is inferred on the lower rate in water movement in $\mathrm{LD}$, that promoted and excess in humidity and a lower quality in the tuberous root.
\end{abstract}

Keywords: tillage, reserve root, soil properties.

\footnotetext{
Recibido: 6 de enero, 2015. Aceptado: 2 de marzo, 2016. Este trabajo formó parte del proyecto grupal titulado "Potencialidad productiva y comercial de la batata (Ipomoea batatas L.) y su relación con la calidad física del suelo", financiado por el Consejo de Desarrollo Científico y Humanístico de la Universidad Central de Venezuela bajo el No PG01-7499-2009, Venezuela.

2 Universidad Central de Venezuela, Facultad de Agronomía, Instituto de Ingeniería Agrícola. Campus Maracay, Maracay, Estado Aragua,Venezuela.Apartado postal 4579.maiby278@gmail.com, aurisgarcia@gmail.com, mariangel8903@hotmail.com
} 


\section{INTRODUCCIÓN}

La batata (Ipomoea batatas (L) Lamb) es un alimento importante en los países en desarrollo, debido a sus sobresalientes características nutricionales con propiedades anticancerígenas y cardiovasculares. Se cultiva a nivel mundial en nueve millones de hectáreas, con una producción anual estimada en 126 millones de toneladas, siendo China el principal productor con 54 millones de toneladas (43\%) (FAOSTAT, 2015).

En Venezuela este cultivo se produce tradicionalmente de forma manual, y mecanizada en pequeñas explotaciones agrícolas, presentan niveles bajos de aplicación tecnológica que impiden una producción masiva, y genera un bajo consumo en comparación con otros rubros dentro de las raíces y tubérculos (Ortega y Marcano, 2000). La incorporación de nuevas prácticas agronómicas en este rubro pretende dar respuesta a la necesidad de ampliar la superficie de explotación, rendimiento y calidad postcosecha, resaltando la importancia de sistemas de labranza, debido a los cambios que provocan en la estructura y calidad del suelo a corto y mediano plazo (Karlen et al., 2003; Dexter, 2004). Así también, entre las prácticas de manejo necesarias para categorizar de manera homogénea la calidad de las raíces, con base en peso, forma, tamaño, raíces sanas, enteras y con menor grado de defectos morfológicos exigidos por los mercados (García y Pacheco, 2008), resulta innovador la incorporación de densidades de siembra distintas a la convencional en combinación a tipos de labranza. Al respecto se considera que, a medida que aumenta el número de plantas por área, incrementa la competitividad entre plantas de los recursos hídricos y nutricionales, resultando raíces tuberosas de menor tamaño, formas regulares y pesos intermedios (Tinedo y Rojas, 2015).

Dentro de los diversos cambios que se derivan de la aplicación de sistemas de labranza, se refieren aquellos que se ejercen sobre los indicadores de calidad postcosecha de la batata como la forma, peso promedio y tamaño de la raíz de reserva (Rós et al., 2012). Asimismo, se refieren efectos indirectos de la disminución en la absorción de agua y nutrientes por el cultivo, y directos de una mayor resistencia mecánica y densidad aparente en suelos degradados por la labranza, sobre la menor elongación radical, peso y rendimiento de la raíz tuberosa de la batata (Agbede y Adekiya, 2009; Abrougui et al., 2014).

Para este estudio se planteó como hipótesis que los sistemas de labranza con arado de disco y cincel, así como el mayor espaciamiento entre plantas del cultivo y la combinación de ambos factores, favorecen el mejoramiento de la condición estructural y propiedades de penetración y circulación de agua en el suelo, permitiendo incrementar el peso y obtener mayor diversidad de tamaños de la batata. Por el contrario, los sistemas de labranza convencional y mínima en combinación con un menor espaciamiento entre plantas del cultivo ejercerán un efecto contrario, aumentando la calidad postcosecha por la mayor preferencia de pesos intermedios, tamaños homogéneos y formas más redondeadas de batata para consumo fresco. Debido a lo anterior, el objetivo de este trabajo fue determinar el efecto de sistemas de labranza y densidades de siembra en la calidad de las raíces tuberosas de la batata.

\section{MATERIALES Y MÉTODOS}

\section{Ubicación de la investigación}

El ensayo se llevó a cabo en el campo experimental de la Sección de Mecanización Agrícola del Instituto de Ingeniería Agrícola de la Facultad de Agronomía, Universidad Central de Venezuela, ubicada en Maracay, Venezuela, en el ciclo de lluvias comprendido entre los meses de junio y diciembre del año 2014. Sus coordenadas son: latitud $10^{\circ} 15^{\prime} 6^{\prime \prime} \mathrm{N}$ y longitud $67^{\circ}$ 36' 5" O, a una altitud de $436 \mathrm{~m}$. Además, se encuentra bajo un clima de bosque seco tropical, registrando una temperatura media anual de $25,5^{\circ} \mathrm{C}$, una estación seca bien definida de cinco a seis meses y una precipitación promedio anual de $834 \mathrm{~mm}$ (INE, 2011). El suelo presenta una pendiente plana cercana a $1 \%$.

\section{Diseño experimental}

El ensayo de campo presentó un arreglo en franjas, considerando a los factores sistemas de labranza (3) y densidad de siembra (2) en el cultivo de la batata. El área general de ensayo presentó $538 \mathrm{~m}^{2}$, subdividido en parcelas representadas por sistemas de labranza 
mecanizada del suelo, ubicados en el sentido de la mayor longitud del lote. Dentro de las parcelas, se establecieron en franjas las distintas densidades de siembra del cultivo con tres repeticiones. Los sistemas de labranza consistieron en labranza vertical profunda con cincel (LC), profunda con arado de disco (LA), media con rastra de disco (LCo) y mínima con rastra de disco (LM). Se utilizó un arado de cincel más tres pases de rastra (LC); un arado de disco más tres pases de rastra (LA); tres pases de rastra (LCo) y un pase de rastra (LM) con cuatro profundidades de trabajo de 18, 23,12 y $7 \mathrm{~cm}$, respectivamente.

Las densidades de siembra correspondieron a 48500 plantas/ha (D1) y 81000 plantas/ha (D2). Las distancias de siembra fueron de $0,25 \mathrm{~cm}$ (D1) y 0,15 $\mathrm{cm}$ (D2) en la hilera y de $0,825 \mathrm{~m}$ entre la hilera de plantas en camellones. Las parcelas de labranza con una dimensión de 22,4 m x 6 m, fueron divididas en franjas para un número de seis unidades experimentales/ parcela. Las unidades experimentales del tratamiento de la combinación sistema de labranza-densidad de siembra (SL-Ds), presentaron una dimensión de 22,4 $\mathrm{m}^{2}$. El número de hileras en cada unidad experimental fue de siete con una longitud de $4 \mathrm{~m}$.

\section{Obtención del material vegetal empleado en la investigación}

Se utilizó la variedad cubana INIVIT B5, caracterizada por ser una raíz comestible de forma redonda a redonda elíptica voluminosa, corteza morada y pulpa crema-amarilla con sabor dulce y agradable. El semillero consistió de tres unidades básicas de riego adyacentes al área de ensayo, consistente de cinco surcos cortos con camellones cada uno. Los mismos presentaron $5 \mathrm{~m}$ de largo con una distancia entre surcos de $1 \mathrm{~m}$, configurados mediante el uso de escardillas. Se hizo un pase de rastra para la preparación del suelo, y el desmalezado fue manual. No se aplicó ningún tipo de producto químico. Se hicieron dos riegos por gravedad en determinadas épocas del cultivo. Los esquejes se sembraron a una distancia de $10 \mathrm{~cm}$ sobre la hilera. El tamaño de los esquejes fue de $20 \mathrm{~cm}$ tanto en semillero, como en el ensayo experimental. El material de siembra se cortó el mismo día de la siembra del ensayo, a 3,5 meses después del establecimiento del semillero.

\section{Muestreo y determinaciones en el suelo y cultivo}

Se recolectaron muestras no disturbadas del suelo con una toma de muestras tipo "Uhland" en cilindros de $5 \times 5 \mathrm{~cm}$ (hxd) a las profundidades de $0-5 \mathrm{~cm}, 5-15 \mathrm{~cm}$ y $15-25 \mathrm{~cm}$ por duplicado, en los tratamientos dentro de la fase de culminación de desarrollo del cultivo (140 días después de la siembra). En los cilindros se determinaron la densidad aparente $\left(\mathrm{Da}, \mathrm{Mg} / \mathrm{m}^{3}\right)$ y espacio poroso de aireación (EPA, $\left.\mathrm{m}^{3} / \mathrm{m}^{3}\right)$ (Pla, 1983; Klute, 1986). Adicionalmente, se tomaron muestras de suelo disturbado a los distintos estratos de profundidad del suelo por triplicado/parcela, para determinar la distribución del tamaño de partículas (DTP, \%) y clase textural de cada estrato (CT), siguiendo el método del hidrómetro (Gee y Bauder, 1986), y el contenido de carbono orgánico $(\mathrm{CO}, \%)$, siguiendo el método de Wakley y Black (1934) empleando espectrometría. Se determinó el nivel de severidad de la degradación del suelo a partir de los valores estandarizados de $\mathrm{Da}$, ETA, RP y CO por estrato, y del perfil de 0-25 cm en los distintos tratamientos según Lal (1994).

Las variables de calidad postcosecha consistieron en longitud y diámetro (L y D) Montaldo (1979), y peso unitario (PU, g) (Echeverría y Rangel, 1992) de las batatas recolectadas en época de cosecha (150 días después de la siembra). Se cuantificaron los porcentajes de batatas que satisficieran simultáneamente los criterios de calidad de L, D y PU, establecidos de preferencia para el consumo directo en los intervalos de largo entre 8 y $13 \mathrm{~cm}$, diámetro entre 7 y $10 \mathrm{~cm}$ y PU entre 100 y 300 g (González, 2014).

Se determinó la forma de la batata mediante el descriptor de forma de Huamán(1992). Adicionalmente, se obtuvo la distribución de frecuencia de los distintos intervalos de PU, L y D, correspondiente a las formas de raíces más encontradas en los tratamientos. La escala de calidad postcosecha, con base en los intervalos de porcentajes, que cumple con los requisitos de aceptación para consumo directo fue: 1 (ninguna) de 0 a 20; 2 (poca) de 21 a 40; 3 (moderada) de 41 a 60; 4 (alta) de 61 a 80; y 5 (muy alta) de 81 a 100. Se aplicó el análisis estadístico descriptivo para las variables del suelo (DTP, Da, EPA, CO, RP) y del cultivo (L, D, PU), y análisis de varianza para un diseño experimental en franjas y por Friedman para las variables mencionadas, a un nivel de significación de 
$\mathrm{p}<0,05$. Se usó la prueba de comparación de medias de Tuckey y el paquete Statistix versión 10.

\section{RESULTADOS Y DISCUSIÓN}

\section{Calidad física del suelo}

Los suelos del ensayo presentaron texturas franco limosa y franco limosa/franco arenosa (Cuadro 1), con una alta fracción de arena muy fina más limo (61 a 74\% $\pm 3,52)$, con baja fracción de arcilla $(1-8 \% \pm 2,54)$ y muy baja de arena media y gruesa (1-2\%) en los distintos estratos de profundidad. De acuerdo con lo mencionado, el suelo presentó una alta susceptibilidad a la degradación estructural por el alto contenido de partículas dispersables en la capa superficial, donde se observaron los mayores cambios asociada a la labranza (Reynolds et al., 2009).

El Cuadro 1 también muestra las propiedades físicas y mecánicas de los suelos afectados por los

Cuadro 1. Efecto de sistemas de labranza y densidades de siembra de batata en las propiedades físicas y mecánicas del suelo. Venezuela. 2014.

Table 1. Soil physical and mechanical properties affected by tillage and seeding density and correspondent levels of soil degradation severity in sweet potato crop. Venezuela. 2014.

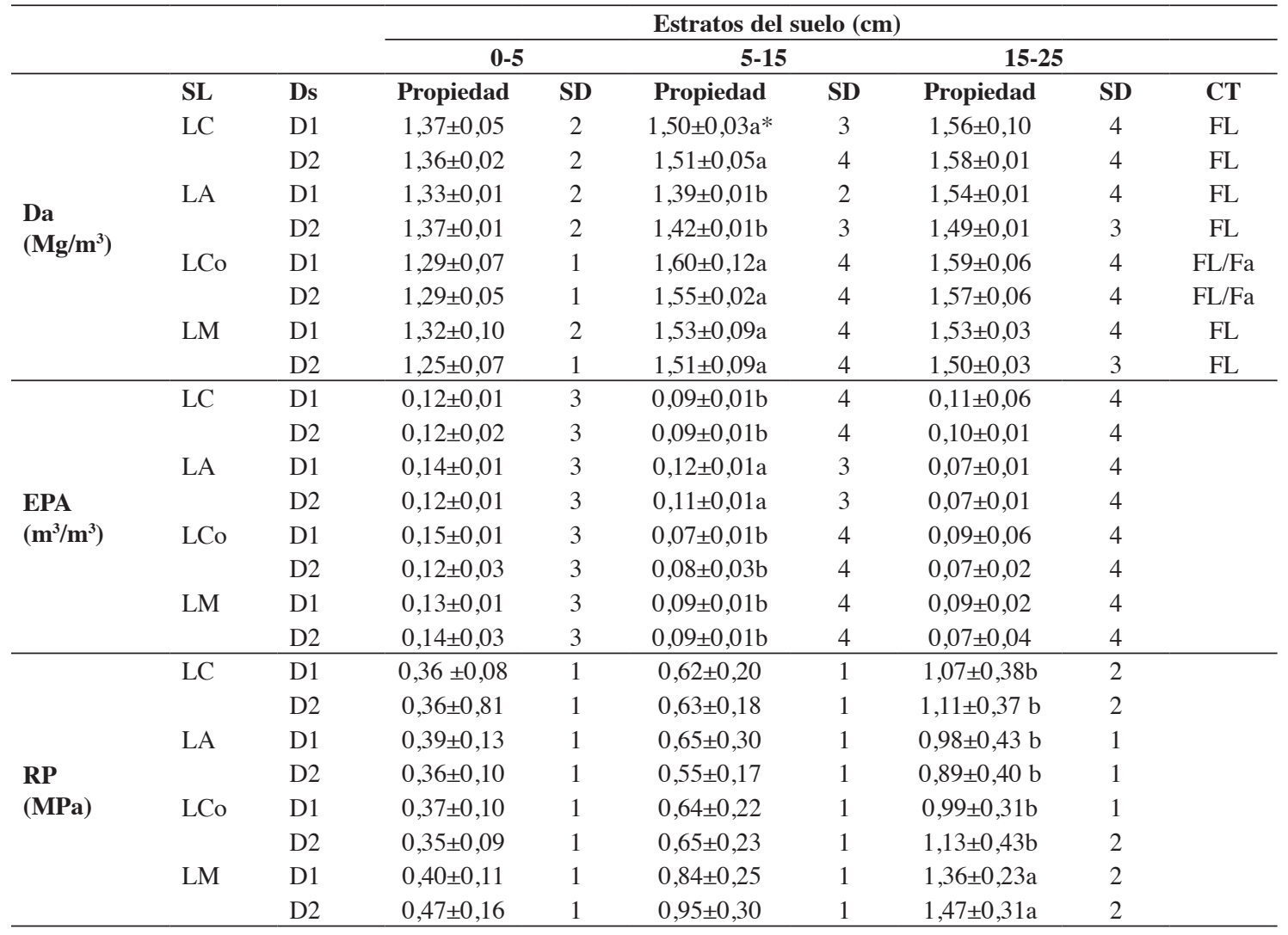

Da: densidad aparente del suelo; EPA: espacio poroso de aireación; RP: resistencia mecánica a la penetración de raíces; SL: sistema de labranza; Ds: densidad de siembra; LC: labranza con cincel; LA: labranza con arado de disco; LCo: labranza convencional; LM: labranza mínima; CT: clase textural de los estratos del suelo; D1: densidad de siembra 1; D2: densidad de siembra 2. SD: nivel de severidad de la degradación 1:ninguna; 2: ligera; 3: moderada; 4: severa y 5: extrema / Da: bulk density; EPA: air filled porosity; RP: mechanical resistance to root penetration; SL: tillage system; Ds: seeding density; LC: tillage with chisel; LA: tillage with disc plow; LCo: conventional tillage; LM: minimum tillage; TC: textural class for the different soil layers; D1: seeding density 1; D2: seeding density 2; SD: soil severity degradation level 1: none; 2: slight; 3: moderate; 4:severe and 5: extreme.

* Letras distintas denotan diferencias significativas $(\mathrm{p} \leq 0,05) /$ Different letters denote statistical differences $(\mathrm{p} \leq 0.05)$. 
tratamientos. De acuerdo con los valores de Da y EPA para estos suelos de textura franco limosa, la condición estructural fue superior al nivel de la superficie del suelo $(0-5 \mathrm{~cm})$, sin presentar limitaciones en aireación, penetración y desarrollo de raíces, con ligero a moderado riesgo de degradación física.

La condición anterior cambia para los estratos a nivel subsuperficial (5-15 y 15-25 cm), los cuales presentaron mayores limitaciones al crecimiento de raíces, como resultado de los niveles de compactación del suelo determinados a partir de los indicadores $\mathrm{Da}$ y EPA, con un riesgo de ligero a severo.

La RP confirmó una mayor dureza del suelo en ambos estratos más profundos. Esta variable se diferenció significativamente $(\mathrm{p} \leq 0,05)$ por efecto de la labranza en el estrato de $15-25 \mathrm{~cm}$, bajo los tratamientos de LM con ligero riesgo a la degradación, al igual que en LCo y LC para este estrato (Cuadro 1). En ninguno de los tratamientos se alcanzó valores de RP limitantes a la penetración de raíces del cultivo
(Lal, 1994; Siegel-Issem et al., 2005). En cuanto a CO (Cuadro 2) se presentó bajo los distintos tratamientos por debajo del límite crítico reportado por Reynolds et al. (2009), establecido en un 2,3\%, que suministra al suelo una alta susceptibilidad a la degradación física por la labranza.

De acuerdo con los niveles críticos de CO (1-3\%) sugeridos por Lal (1984), el suelo bajo los distintos tratamientos presentó en general moderadas limitaciones a la pérdida de la estructura y de la capacidad de retención de agua y nutrientes. No se observaron diferencias significativas por Friedman $(p>0,05)$ para $\mathrm{CO}$ entre los sistemas de labranza y densidades de siembra.

En relación con la severidad de la degradación física determinada según las variables Da, EPA, CO y RP para los distintos estratos (Cuadro 1), se revelaron niveles de ninguna a moderada severidad de la degradación en el estrato superficial del suelo $(0-5 \mathrm{~cm})$, y de ninguna a severa en los estratos más

Cuadro 2. Efecto de sistemas de labranza y densidades de siembra de batata en el carbono orgánico del suelo. Venezuela. 2014.

Table 2. Soil organic carbon under different tillage systems and seeding densities in sweet potato crop and correspondent levels of soil degradation severity. Venezuela. 2014.

\begin{tabular}{lccccccc}
\hline & & \multicolumn{6}{c}{ Estratos del suelo (cm) } \\
\cline { 2 - 8 } \multicolumn{2}{c}{ Tratamiento } & \multicolumn{2}{c}{$\mathbf{0 - 5}$} & \multicolumn{2}{c}{$\mathbf{5 - 1 5}$} & \multicolumn{1}{c}{$\mathbf{1 5 - 2 5}$} \\
\hline SL & Ds & CO $(\boldsymbol{\%})$ & SD & CO (\%) & SD & CO (\%) & SD \\
\hline LC & D1 & 1,71 & 3 & 1,09 & 3 & 1,03 & 3 \\
& D2 & 1,97 & 3 & 1,28 & 3 & 1,25 & 3 \\
LA & D1 & 1,68 & 3 & 1,46 & 3 & 1,15 & 3 \\
& D2 & 2,00 & 3 & 1,62 & 3 & 1,48 & 3 \\
LCo & D1 & 1,65 & 3 & 1,25 & 3 & 1,00 & 3 \\
& D2 & 1,96 & 3 & 1,40 & 3 & 1,18 & 3 \\
LM & D1 & 2,01 & 3 & 1,33 & 3 & 1,26 & 3 \\
& D2 & 1,94 & 3 & 1,35 & 3 & 0,97 & 3 \\
\hline
\end{tabular}

SL: sistema de labranza; Ds: densidad de siembra; CO: carbono orgánico del suelo; LC: labranza con cincel; LA: labranza con arado de disco; LCo: labranza convencional; LM: labranza mínima; D1: densidad de siembra 1; D2: densidad de siembra 2. SD: nivel de severidad de la degradación 1: ninguna; 2 : ligera; 3 : moderada; 4 : severa y 5 : extrema / SL: tillage system; Ds: seeding density; CO: soil organic carbon; LC: tillage with chisel; LA: tillage with disc plow; LCo: conventional tillage; LM: minimum tillage; D1: seeding density 1 ; D2: seeding density 2; SD: soil severity degradation level 1 : none; 2 : slight; 3 : moderate; 4: severe and 5: extreme. 
profundos, presentando los tratamientos de LA la mejor condición estructural respecto a LCo, LC y LM, a nivel de $5-15 \mathrm{~cm}$ del suelo, con ninguno a moderado riesgo a la degradación física en este estrato. Esto mismo lo refleja el promedio de los indicadores (PIE) calculado por estrato en los distintos tratamientos (Figura 1), el cual fue menor para el estrato superficial y mayor para los estratos más profundos. Así también, el PIE fue menor con LA en el estrato intermedio, que evidencia una mejor condición estructural del suelo en profundidad con este sistema de labranza.

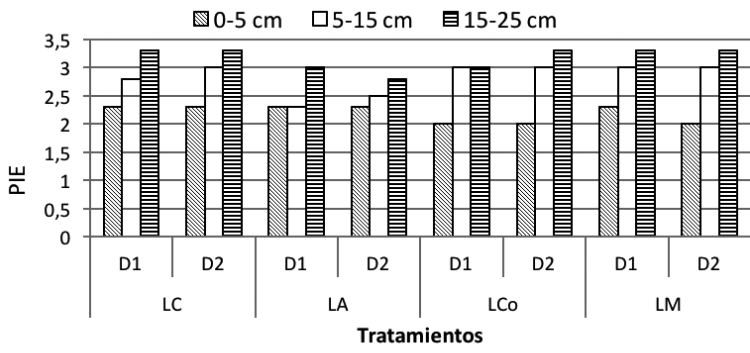

Figura 1. Efecto de sistemas de labranza y densidades de siembra de batata en el promedio de los indicadores estructurales por estrato de suelo. Venezuela. 2014 LC: labranza con cincel; LA: labranza con arado de disco; LCo: labranza convencional; LM: labranza mínima; D1: densidad de siembra 1; D2: densidad de siembra 2. PIE: promedio de los indicadores estructurales por estrato de suelo.

Figure 1. Structural indexes average (PEI) by soil layer under different tillage systems and seeding densities in sweet potato crop. Venezuela. 2014.

LC: tillage with chisel; LA: tillage with disc plow; LCo: conventional tillage; LM: minimum tillage; D1: seeding density 1; D2: seeding density 2. PIE: average of structural indexes for each soil layer.

El efecto del rompimiento de capas compactadas y volteado del suelo en profundidad significó una profundidad efectiva superior en LA (desde 0-15 $\mathrm{cm})$. Cabe destacar, que los estratos presentaron en su mayoría un ligero a moderado riesgo a la degradación (PIE:2 a 3,3), sin contrastes importantes entre los mismos, destacándose la condición física y mecánica del suelo ligeramente superior en los tratamientos de LA a nivel del estrato intermedio.

\section{Calidad postcosecha del cultivo}

Los PU correspondiente a las formas de raíces más encontradas en los distintos tratamientos, se presentaron entre 183 y 252 g (Figura 2), considerándose raíces tuberosas medianas (150 a $250 \mathrm{~g}$ ) (Castillo et al., 2014; González, 2014).

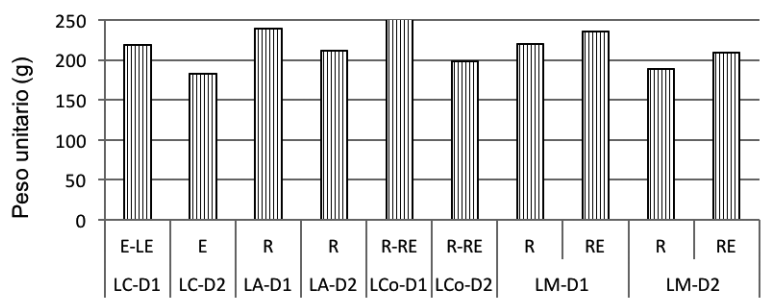

Formas de la raíz más encontradas en los tratamientos

Figura 2. Efecto de sistemas de labranza y densidades de siembra de batata en los pesos unitarios de raíces tuberosas. Venezuela. 2014.

LC: labranza con cincel; LA: labranza con arado de disco; LCo: labranza convencional; LM: labranza mínima; D1: densidad de siembra 1; D2: densidad de siembra 2. E: elíptica: LE: largo elíptica; R: redonda; RE: redonda elíptica.

Figure 2. Unitary weigh of sweet potato reserve roots under different tillage systems and seeding densities. Venezuela. 2014.

LC: tillage with chisel; LA: tillage with disc plow; LCo: conventional tillage; LM: minimum tillage; D1: seeding density 1 ; D2: seeding density $2 ; \mathrm{E}$ : elliptical; LE: long elliptical; R: rounded; RE: rounded elliptical.

No se encontraron diferencias estadísticamente significativas $(\mathrm{p} \geq 0,05)$ entre los tratamientos para PU. En tal sentido, los sistemas de labranza profunda (arado y cincel) y convencional no se diferenciaron respecto a la labranza mínima. Al respecto, se señala que el menor peso fresco de la raíz de reserva y mayor número de raíces por planta en el sistema de labranza convencional al comparar con la labranza mínima, derivaron en un mayor rendimiento del cultivo en el sistema convencional (Rós et al., 2013). Las ventajas de los primeros sistemas (profunda y convencional) se basan en la reducción de la compactación del suelo en 
épocas tempranas de desarrollo del cultivo (0-65 dds) y en el mayor impacto positivo sobre el rendimiento del cultivo (Sajjapongse y Roan, 1982; Anikwe y Ubochi, 2007), al propiciarse en el periodo inicial (entre dos a ocho semanas) un alto número de raíces por planta y su desarrollo posterior como raíces de reserva (Henderson, 2003).

El PU considerado mediano se atribuyó a la alta concentración de la precipitación en el ciclo del cultivo (860 mm,92\% del total), y a su interacción con el suelo de textura franco limosa de muy baja pendiente (alrededor de $1 \%$ ), que propiciaron un inadecuado balance aguaaire y afectaron al cultivo en el periodo de iniciación de raíces y tuberización. En este sentido, suelos de textura pesada y pobremente drenados, susceptibles a frecuente anegamiento y pobre aireación, impiden el crecimiento de las raíces de reserva de batata, reduciendo su tamaño y rendimiento (Musembi, 2013), además de conllevar al deterioro de la calidad postcosecha, color atípico de la pulpa y menores niveles de peso seco y azúcar simple de las raíces, entre otros (Henderson, 2003).

De manera general, los PU de batata bajo los distintos tratamientos se presentaron con mayor frecuencia en los grupos I, II y III (66 a 100\%), que resultan de preferencia a nivel de los consumidores directos (100 a $300 \mathrm{~g})$. En este sentido, Valiente et al. (2010) establecieron un intervalo de PU entre $200 \mathrm{y}$ $350 \mathrm{~g}$ como de mayor calidad para batata; mientras que Segura et al. (2003) consideraron que esta categoría corresponde al intervalo de 150 a $450 \mathrm{~g}$ para una diversidad de raíces y tubérculos. En cuanto a la menor relación porcentual de frecuencia de PU superiores a los 260 g con tendencia a $400 \mathrm{~g}$, se atribuyó a que las raíces y tubérculos, por su forma de crecimiento y desarrollo, presentan una variación de pesos como características intrínsecas dentro del racimo abierto de formación de raíces tuberosas (Tinedo y Rojas, 2015).

En relación a la forma de las batatas, los distintos intervalos revelaron la predominancia de batatas más largas y delgadas con formas elíptica y largo elíptica (48 a 79\%) en comparación a las formas redonda y redonda elíptica (21-33\%) en los tratamientos de LC; con tendencia redondeada (redonda y redonda elíptica) (58-71\%) en comparación con la forma elíptica y largo elíptica (29-37\%) en los tratamientos de Lco y LM, y mucho más redonda (50-51\%) que elíptica y largo elíptica (25-31\%) en LA.

La raíz de reserva de batata responde con alteraciones de la forma al limitarse su crecimiento en longitud, debido a la alta densidad aparente y resistencia a la penetración de los estratos subsuperficiales del suelo, presentando formas más alargadas bajo labranza convencional y más achatadas en suelos compactados bajo labranza reducida (Rós et al., 2012; Abrougui et al., 2014). De manera similar, las raíces absorbentes de distintos cultivos manifiestan deformaciones a partir del engrosamiento, desviaciones en la dirección vertical y concentración de raíces a nivel muy superficial del suelo, cuando se superan los valores críticos de densidad aparente y resistencia mecánica (Hajabbasi, 2001; Gregory, 2006; Reinert et al., 2008). Lo citado anteriormente, no es consistente con las variaciones de forma de la raíz encontradas, se esperaba más alargadas en LA, como respuesta al menor grado de limitación física y mecánica del suelo. Esta característica física no uniforme puede deberse a la falta de selección y certificación de los materiales utilizados de raíces tuberosas para las siembras comerciales (García y Pacheco, 2008).

El análisis de la calidad postcosecha para consumo directo según PU (Figura 3), permitió determinar que todos los tratamientos concentraron una alta cantidad de

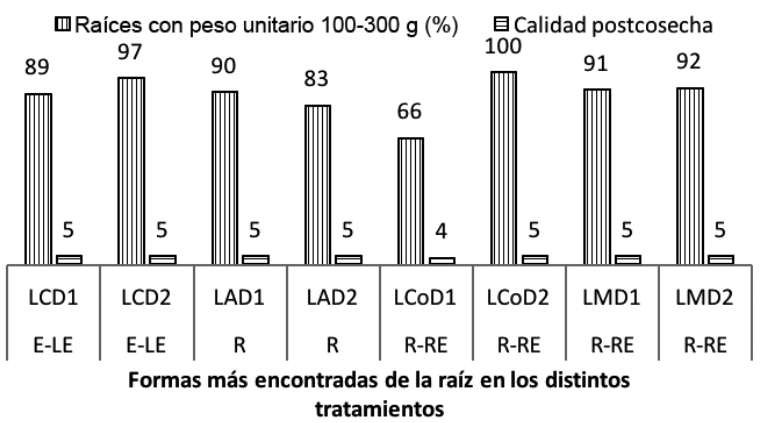

Figura 3. Efecto de sistemas de labranza y densidades de siembra de batata sobre el porcentaje de raíces tuberosas con pesos unitarios de preferencia y niveles de calidad postcosecha. Venezuela. 2014. LC: labranza con cincel; LA: labranza con arado de disco; LCo: labranza convencional; LM: labranza mínima; D1: densidad de siembra 1; D2: densidad de siembra 2. E: elíptica: LE: largo elíptica; R: redonda; RE: redonda elíptica.

Figure 3. Percentage of sweet potato reserve roots with unitary weigh of preference and levels of postharvest quality under different tillage systems and seeding densities. Venezuela. 2014.

LC: tillage with chisel; LA: tillage with disc plow; LCo: conventional tillage; LM: minimum tillage; D1: seeding density 1; D2: seeding density 2; E: elliptical; LE: long elliptical; R: rounded; RE: rounded elliptical. 
raíces en el intervalo de preferencia muy alta (80-100\%), excepto LCo-D1 con la menor calidad postcosecha (alta), por presentar PU superiores a $300 \mathrm{~g}$ (34\%).

El mayor porcentaje de raíces con la mejor calidad comercial con base en las variables L y D, se presentó a partir de la aplicación de LA-D1 seguido por los tratamientos de LCo, y en menor grado los restantes (Figura 4).

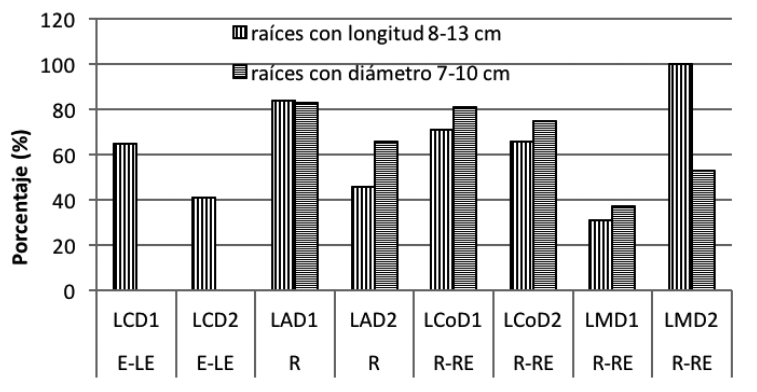

Formas más encontradas de la raíz en los distintos tratamientos

Figura 4. Efecto de sistemas de labranza y densidades de siembra de batata sobre el porcentaje de raíces tuberosas, con tamaños de preferencia. Venezuela, 2014.

LC: labranza con cincel; LA: labranza con arado de disco; LCo: labranza convencional; LM: labranza mínima; D1: densidad de siembra 1; D2: densidad de siembra 2. E: elíptica: LE: largo elíptica; R: redonda; RE: redonda elíptica.

Figure 4. Percentage of sweet potato reserve roots with sizes of preference under different tillage systems and seeding densities.Venezuela, 2014.

LC: tillage with chisel; LA: tillage with disc plow; LCo: conventional tillage; LM: minimum tillage; D1: seeding density 1 ; D2: seeding density 2; E: elliptical; LE: long elliptical; R: rounded; RE: rounded elliptical.

La calidad postcosecha según el tamaño (Figura 5) que correspondió a los niveles no aceptable y poco aceptable se obtuvo en los tratamientos que incorporaron la labranza con cincel y la labranza mínima (LM-D1), respectivamente. Las raíces fueron muy poco engrosadas y más largas en los tratamientos de LC, y poco engrosadas y muy cortas en LM-D1. La más alta aceptación correspondió a LA-D1, seguido por los tratamientos de LCo.

En referencia a la calidad postcosecha que integra las variables anteriores (PU-L-D) (Figura 5) se

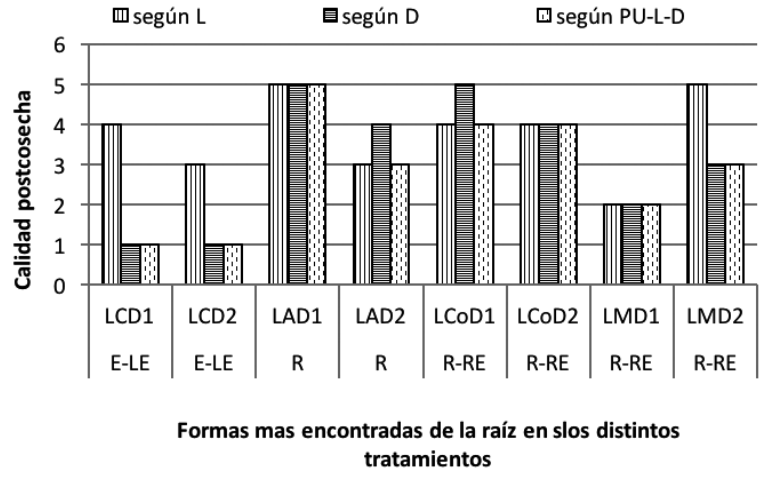

Figura 5. Efecto de sistemas de labranza y densidades de siembra de batata sobre el nivel de calidad postcosecha de raíces tuberosas.Venezuela. 2014.

LC: labranza con cincel; LA: labranza con arado de disco; LCo: labranza convencional; LM: labranza mínima; D1: densidad de siembra 1; D2: densidad de siembra 2. E: elíptica: LE: largo elíptica; R: redonda; RE: redonda elíptica; PU: peso unitario; L: longitud; D: diámetro.

Figure 5. Level of postharvest quality of sweet potato roots under different tillage systems and seeding densities. Venezuela. 2014.

LC: tillage with chisel; LA: tillage with disc plow; LCo: conventional tillage; LM: minimum tillage; D1: seeding density 1 ; D2: seeding density 2; E: elliptical; LE: long elliptical; R: rounded; RE: rounded elliptical; PU: unitary weight; L: length; D: diameter.

encontró que en el tratamiento LA-D1 se obtuvo muy alta aceptación para consumo directo, seguido por los tratamientos de LCo (alta aceptación), LA-D2 y LMD2 (moderadamente aceptable), y por LC y LM-D1 (no aceptable y poco aceptable). Las limitaciones a la calidad postcosecha del cultivo estuvieron dadas principalmente por no satisfacerse las dimensiones requeridas, tanto de largo como de diámetro, por lo cual, ambas variables determinaron finalmente la calidad postcosecha del cultivo.

\section{Influencia de la severidad de la degradación del suelo sobre la calidad postcosecha del cultivo de la batata}

La mayor coincidencia entre los grupos de tratamientos de más baja calidad, en ambos sentidos, estuvo representada por LMD1, LCD1 y LCD2 (Figura 6). En este grupo el valor comercial no 


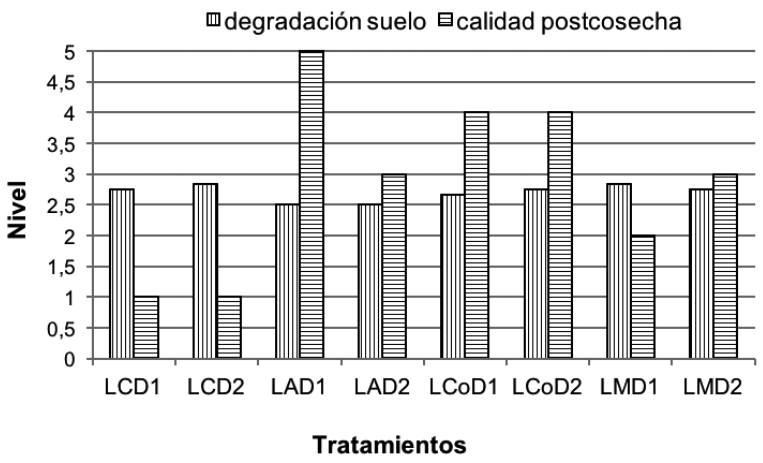

Figura 6. Efecto de sistemas de labranza y densidades de siembra en la calidad postcosecha de la batata y el nivel de severidad de la degradación del suelo en el estrato de 0- $25 \mathrm{~cm}$. Venezuela, 2014.

LC: labranza con cincel; LA: labranza con arado de disco; LCo: labranza convencional; LM: labranza mínima; D1: densidad de siembra 1; D2: densidad de siembra 2; Cv: nivel de aceptación postcosecha del cultivo de acuerdo a cada variable: 1 (ninguna); 2 (poca); 3 (moderada); 4 (alta); 5 (muy alta); CP: nivel de aceptación postcosecha del cultivo: 1 (ninguna); 2 (poca); 3 (moderada); 4 (alta); 5 (muy alta); PIE: promedio de los indicadores estructurales en el estrato de $0-25 \mathrm{~cm}$ del suelo.

Figure 6. Postharvest quality and soil degradation severity level under different tillage systems and seeding densities in sweet potato crop. Venezuela, 2014.

LC: tillage with chisel; LA: tillage with disc plow; LCo: conventional tillage; LM: minimum tillage; D1: seeding density 1; D2: seeding density 2; Cv: postharvest quality level of acceptance according to each variable: 1 (none); 2 (slight); 3 (moderate); 4 (high); 5 (very high); CP: postharvest level of acceptance: 1 (none); 2 (slight); 3 (moderate); 4 (high) and 5 (very high); PIE: average of structural indexes for $0-25 \mathrm{~cm}$ soil layer.

aceptable de la batata, vinculado a las limitaciones al dimensionamiento de la raíz de reserva, contrastó con el grado de severidad a la degradación en el estrato de 0-25 cm de estos suelos (PIE: 2,8), el cual se observó muy similar al resto de los tratamientos (PIE: 2,5-2,8).

Destaca el grado de severidad a la degradación en el perfil de $0-25 \mathrm{~cm}$ inferior en los tratamientos de LA y LCo-D1 (PIE: 2,5-2,7), con calidad comercial muy alta a moderada y, el riesgo a la degradación superior en LM-D1 y LC $(2,8)$ con calidades no aceptable a poco aceptable de la raíz reservante de batata para consumo fresco (Figura 6). A pesar de lo encontrado, no es posible explicar la falta de idoneidad para producir batatas de calidad comercial en los tratamientos con labranza mínima bajo la distancia de siembra más larga y con cincel, basada en la gama de cualidades del suelo consideradas.

Se presume la presencia de variaciones de la humedad del suelo en el estrato de crecimiento de las raíces durante la tuberización, asociadas a la labranza, la alta precipitación en el ciclo de cultivo y la textura franco-limosa del suelo con relieve plano. Si el suelo es muy fértil, pesado y húmedo los rendimientos son muy bajos al igual que su calidad; las raíces de batata de mejor calidad se obtienen en suelos arenosos y pobres (Anikwe y Ubochi, 2007).

Lo anteriormente mencionado, pudo contribuir a una mayor sensibilidad a cambios de forma y tamaño de las raíces en los distintos tratamientos. De estos tratamientos, la labranza con cincel que implicó el rompimiento vertical del suelo, pudo no favorecer el drenaje interno rápido en la etapa vegetativa y de formación de tubérculos, limitando con ello el engrosamiento de la raíz de reserva. Por el contrario, una posible mayor tasa de penetración y movimiento de agua en los tratamientos con arado y convencional (que implicaron una mayor alteración del suelo en el estrato de labranza), pudo favorecer el crecimiento de órganos vegetativos y el engrosamiento de la batata en la etapa de tuberización, dando lugar a formas más redondeadas y pesos medianos de preferencia (Rós et al., 2013).

Al respecto, se infiere que la alta rugosidad y acumulación de agua en las microdepresiones de la superficie del suelo establecidas por el arado de disco, incrementó la infiltración de agua en época temprana del cultivo con persistencia en etapas tardías (Vidal y Paz, 2003). Por otra parte, los trabajos en labranza mínima son escasos y controversiales; no obstante, se refiere el efecto integrado de las propiedades físicas sobre la expresión de una alta resistencia mecánica del suelo limitando la elongación de la raíz y dando lugar a raíces más achatadas (Rós et al., 2012).

\section{AGRADECIMIENTO}

Se agradece el financiamiento del Consejo de Desarrollo Científico Humanístico ( $\mathrm{CDCH})$ de la Universidad Central de Venezuela, a través del proyecto grupal PG-7449-09. 


\section{LITERATURA CITADA}

Abrougui K., S.H.H. Chehaibi, I. Boukhalfa, B. Chenini, Douh, and M. Nemri. 2014. Soil bulk density and potato tuber yield as influenced by tillage systems and working depths. Greener J. Agric. Sci. 4:046-051.

Agbede, T.M., and A.O. Adekiya. 2009. Tillage effects on soil properties and performance of sweet potato on an Alfisol in Southwestern Nigeria. Eur. J. Sustain. Agric. 3:561-568.

Anikwe, M.A.N., and J.N. Ubochi. 2007. Short-term changes in soil properties under tillage systems and their effect on sweet potato (Ipomea batatas L.) growth and yield in an Ultisol in south-eastern Nigeria. Austr. J. Soil Res. 45:351-358.

Castillo, M.,A. Brenes, P. Esker, y L. Gómez. 2014. Evaluación agronómica de trece genotipos de camote (Ipomoea batatas L.). Agron. Costarricense 38(2):67-81.

Dexter, A. 2004. Soil physical quality. Part I. Theory, effects of soil texture, density, and organic matter, and effects on root growth. Geoderma 3-4:201-214.

Echeverría, H., y O. Rangel. 1992. Caracterización físico mecánica de algunos productos hortofrutícolas, En: R. Dávila, y H. Echeverría, editores, Taller de transferencia de manejo y tecnología postcosecha. Jornadas Técnicas de Ingeniería Agrícola. Universidad Central de Venezuela, Maracay, VEN. p. 10-20.

FAOSTAT 2015. FAO statistical databases. http://faostat3. fao.org/browse/Q/QC/E (consultado 7 nov. 2015).

García, A., y E. Pacheco. 2008. Caracterización postcosecha del apio criollo cultivado en el municipio Tovar, estado Mérida-Venezuela. Rev. Agron. Trop. 58:409-416.

Gee, R., and P. Bauder. 1986. Particle size. In: A. Klute, editor, Methods of soil analysis. Part 1. $2^{\text {nd }}$ ed. Agron. Monogr. N. 9. ASA y SSSA, Madison, WI, USA. p. 443-461.

González, Z. 2014. Evaluación de indicadores de calidad postcosecha en la raíz de batata (Ipomoea batatas (L.) Lamb) cultivadas en un sistema de siembra convencional, Tesis grado, UCV, Maracay, VEN.

Gregory, P. 2006. Plant roots, growth, activity and interactions with soils. Blackwell Publishing, Garsington Road, Oxford, GBR.

Hajabbasi, M.A. 2001. Tillage effects on soil compactness and wheat root morphology. J. Agric. Sci. Technol. 3:67-77.

Henderson, C. 2003. Literature review from rural water use efficiency. Project 2003. In: Horticultural Australia Ltd, editor, Developing smooth skinned easy to peel sweet potatoes Final Report HAL project. Queensland
Government DPI \& F, Queensland, Australia. p. 1-8. http://ausveg.com.au/intranet/technical-insights/docs/ VG02114_complete.pdf. (accesed 13 dic. 2015).

Huamán, Z. 1992. Descriptores de la batata. Centro Internacional de la Papa, PER.

INE (Instituto Nacional de Estadística). 2011. Informe geoambiental 2011. Estado Aragua. http://www.ine. gov.ve/documentos/Ambiental/PrincIndicadores/pdf/ Informe_Geoambiental_Aragua.pdf. (consultado 7 nov. 2015).

Karlen, D., C. Ditzler, and S. Andrews. 2003. Soil quality: why and how?. Geoderma 114:145-156.

Klute, A. 1986. Water relations: Laboratory methods. In: A. Klute, editor, Methods of soil analysis. Part $1.2^{\text {nd }}$ ed. Agron. Monogr. No. 9. ASA y SSSA, Madison, WI, USA. p. 635-662.

Lal, R. 1994. Methods and guidelines for assessing sustainable use of soil and water resources in the tropics. Soil Conservation Service Technical Monograph, No. 21. USDA, USA.

Montado, A. 1979. La yuca o mandioca. Instituto Interamericano de Ciencias Agrícolas (IICA), SanJosé, CRC.

Musembi, B. 2013. Breeding sweet potato (Ipomoea batatas [L.] Lam.) for drought tolerance in Kenya. Thesis PhD., University of Nairobi, KEN.

Ortega, E., y J. Marcano. 2000. Fortalezas del proceso productivo de la batata, Revista FONAIAP Divulga No 66. http://sian.inia.gob.ve/repositorio/revistas_tec/ FonaiapDivulga/fd66/texto/batata.htm (consultado 5 oct. 2015)

Pla, I. 1983. Metodología para la caracterización física con fines de diagnóstico de problemas de manejo y conservación de suelos en condiciones tropicales. Rev. Fac. Agron. (UCV) Alcance 32:91.

Reynolds W.D., C.F. Drury, C.S. Tan, C.A. Fox, and X.M. Yang. 2009. Use of indicators and pore volumefunction characteristics to quantify soil physical quality. Geoderma 152:252-263.

Reinert, D. J., J.A. Albuquerque, J.M. Reichert, C.A. Martín, and M.C. Andrada. 2008. Bulk density critical limits for normal root growth of cover crops. Rev. Bras. Ciênc. Solo 32:1805-1816.

Rós, A.B., J. Tavares-Filho, e G.M. Barbosa. 2012. Propriedades físicas de solo e crescimento de batatadoce em diferentes sistemas de preparo. R. Bras. Ciênc. Solo 37:242-250.

Rós, A.B., J. Tavares-Filho, e G.M. Barbosa. 2013. Produtividade da cultura da batata-doce em diferentes 
sistemas de preparo do solo. Bragantia, Campinas 72:140-145.

Sajjapongse, A., and Y.C. Roan. 1982. Physical factors affecting root yield of sweet potato (Ipomoea batatas (L) Lam). In: L. Villareal, and T.D. Griggs, editors, Sweet potato: proceedings of the first international symposium, Asian Vegetable Research and Development Centre, Shanhua, TWN. p. 203-208.

Segura, A., D. Saborío, y M. Sáenz. 2003. Algunas normas de calidad en raíces y tubérculos tropicales de exportación de Costa Rica. Agron. Costarricense 27(1):49-61.

Tinedo, R., y M.G. Rojas. 2015. Evaluación de la calidad postcosecha en la raíz tuberosa de batata (Ipomoea batatas L. var. Cubana) cultivada en cuatro sistemas de labranza y dos densidades de siembra. Tesis de grado, Universidad Central de Venezuela, Maracay, VEN.

Siegel-Issem, C., J. Burger, R. Powers, F. Ponder, and S. Patterson. 2005. Seedling root growth as a function of soil density and water content. Soil Sci. Soc. Am. J. 69:215-226.

Valiente, J.O., A.M. Jeaume, L.S. Castelli, L. Sila, y C. Barreto. 2010. Proceso organizacional de productores de batata, entre ríos (Mayo 2010). Experiencia de asociativismo con productores de batata. http://aader. org.ar/XV_Jornada/trabajos/espanol/Estrategias_y_ experiencias/Experiencia/Trabajo\%20112\%20 Completo.pdf. (consultado 1 nov. 2015).

Vidal, E., y A. Paz. 2003. Evolución del microrrelieve de la superficie del suelo medido a diferentes escalas. En: J. Álvarez-Benedi, y P. Marinero, editores, Estudios de la zona no saturada del suelo. Vol. VI. Universidad de la Coruña, ES. p. 203-208.

Walkley, A., and I. Black.1934. An examination of the Degtjareff method for determining soil organic matter and a proposed modification of the chromic acid titration method. Soil Sci. 37:29-38. 
